\title{
Measuring airway dimensions during bronchoscopy using anatomical optical coherence tomography
}

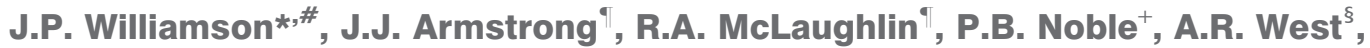 \\ S. Becker ${ }^{\ddagger}$, A. Curatolo ${ }^{\Uparrow}$, W.J. Noffsinger*, H.W. Mitchell ${ }^{\S}$, M.J. Phillips ${ }^{f}$, \\ D.D. Sampson", D.R. Hillman*,** and P.R. Eastwood*,\#,**
}

ABSTRACT: Airway dimensions are difficult to quantify bronchoscopically because of optical distortion and a limited ability to gauge depth. Anatomical optical coherence tomography (aOCT), a novel imaging technique, may overcome these limitations. This study evaluated the accuracy of aOCT against existing techniques in phantom, excised pig and in vivo human airways.

Three comparative studies were performed: 1 ) micrometer-derived area measurements in 10 plastic tubes were compared with aOCT-derived area; 2) aOCT-derived airway compliance curves from excised pig airways were compared with curves derived using an endoscopic technique; and 3) airway dimensions from the trachea to subsegmental bronchi were measured using aOCT in four anaesthetised patients during bronchoscopy and compared with computed tomography (CT) measurements.

Measurements in plastic tubes revealed aOCT to be accurate and reliable. In pig airways, aOCTderived compliance measurements compared closely with endoscopic data. In human airways, dimensions measured with $\mathrm{OOCT}$ and CT correlated closely. Bland-Altman plots showed that aOCT diameter and area measurements were higher than CT measurements by $7.6 \%$ and $15.1 \%$, respectively.

Airway measurements using aOCT are accurate, reliable and compare favourably with existing imaging techniques. Using aOCT with conventional bronchoscopy allows real-time measurement of airway dimensions and could be useful clinically in settings where knowledge of airway calibre is required.

KEYWORDS: Airway dimensions, computed tomography, interventional bronchoscopy, optical coherence tomography, quantitative bronchoscopy

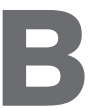
ronchoscopy is a widely performed procedure with many indications. Several of these, such as airway stenosis, tracheomalacia and the deployment of valves for endoscopic lung volume reduction, require knowledge of airway dimensions in order to optimise the interventions and/or to assess disease progression longitudinally. To date, bronchoscopists have relied on pre-procedure imaging, usually with computed tomography (CT), to quantify airway dimensions such as calibre or length of stenosis. CT has a proven capacity to provide such measurements, but it cannot be utilised during a procedure. This presents a major limitation when, for example, during a stenting procedure the bronchoscopist wishes to confirm the stenosis dimensions to ensure selection of an appropriate stent, as complications can arise from poorly sized stents $[1,2]$.

While it is possible to derive quantitative information directly from bronchoscopic images, two major impediments make doing so difficult. First, bronchoscopes display three-dimensional anatomy as a two-dimensional image, thereby limiting the ability of an observer to gauge depth. Secondly, the wide-angle lens at the tip of the bronchoscope, while employed to facilitate the widest possible view, results in image distortion, particularly at the peripheries. Several techniques have been developed to permit quantification from bronchoscopic images [3-9]. However, these techniques entail complex correction algorithms, and for accurate calibration often require targets

\section{AFFILIATIONS}

*Dept of Pulmonary Physiology, Sir Charles Gairdner Hospital,

${ }^{f}$ Dept of Respiratory Medicine, Sir Charles Gairdner Hospital,

\#School of Anatomy \& Human

Biology, University of Western Australia,

"Optical+Biomedical Engineering Laboratory, School of Electrical,

Electronic \& Computer Engineering, University of Western Australia,

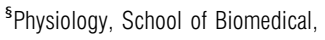
Biomolecular and Chemical, Sciences, University of Western Australia,

${ }^{+}$Division of Clinical Sciences, Telethon Institute for Child Health Research, and

**West Australian Sleep Disorders Research Institute, Perth, Australia.

\section{CORRESPONDENCE}

J.P. Williamson

Dept of Pulmonary Physiology, Level

5, G-Block

Sir Charles Gairdner Hospital

Perth

Western Australia

Australia

E-mail: Jonathan.Williamson@

health.wa.gov.au

Received:

March 142009

Accepted after revision:

May 232009

First published online:

June 182009 
a)
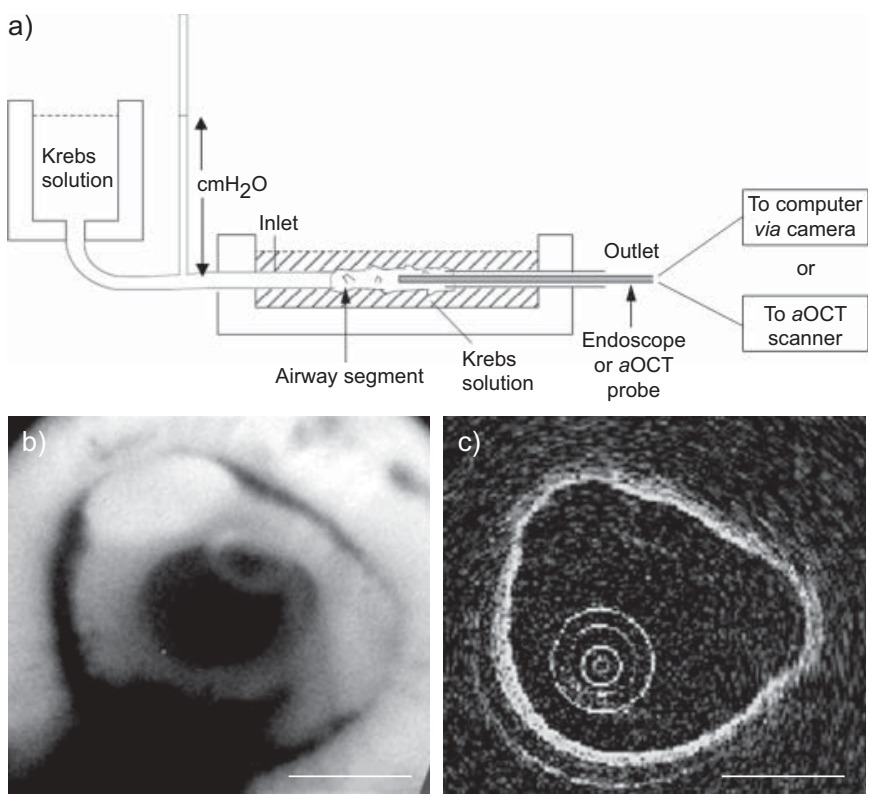

FIGURE 1. a) The arrangement of the airway segments in the organ chamber. The endoscope or anatomical optical coherence tomography (aOCT) probe is inserted through the outlet channel into the airway lumen. Corresponding internal endoscopic (b) and aOCT (c) airway views are shown. Note that within the aOCT cross-section, the innermost circle is the reflection from the probe itself while the outer two circles are reflections from the inner and outer walls of the plastic catheter. The thickness of the white line in the aOCT view (the airway wall) represents penetration of light into the submucosal tissue. Scale bars=3 mm. Modified from [19].

of known size (e.g. biopsy forceps or measuring devices) to be placed in the field of view.

This paper describes a novel imaging technique that addresses many of these problems. Optical coherence tomography (OCT) is a light-based imaging technique based on the principle of lowcoherence interferometry. In its conventional form, OCT allows subsurface airway analysis to a depth of $2-3 \mathrm{~mm}$, providing an "optical biopsy" approaching histological resolution. Respiratory applications focus on the detection of malignant and dysplastic epithelial changes [10, 11]. Anatomical OCT (aOCT) is a modification of conventional OCT designed to range over larger distances to permit real-time macroscopic imaging of hollow organs [12]. Applications in the human upper airway demonstrate the in vivo utility of this technique, but it has yet to be validated in the tracheobronchial tree [13-16].

Our group has previously detailed the technical considerations of applying $a \mathrm{OCT}$ to the central airways [17]. The purpose of this paper is to validate $a \mathrm{OCT}$ for use in the human tracheobronchial tree.

\section{METHODS}

The accuracy of $a \mathrm{OCT}$ was systematically validated in plastic tubes, pig bronchi and human airways by comparing measurements made with $a \mathrm{OCT}$ and other established imaging techniques. The Human Research Ethics Committee at our hospital approved this study and patients provided informed consent. The animal study was approved by our institutional Animal Ethics Committee. Further details of the following methods can be found in an accompanying online repository.
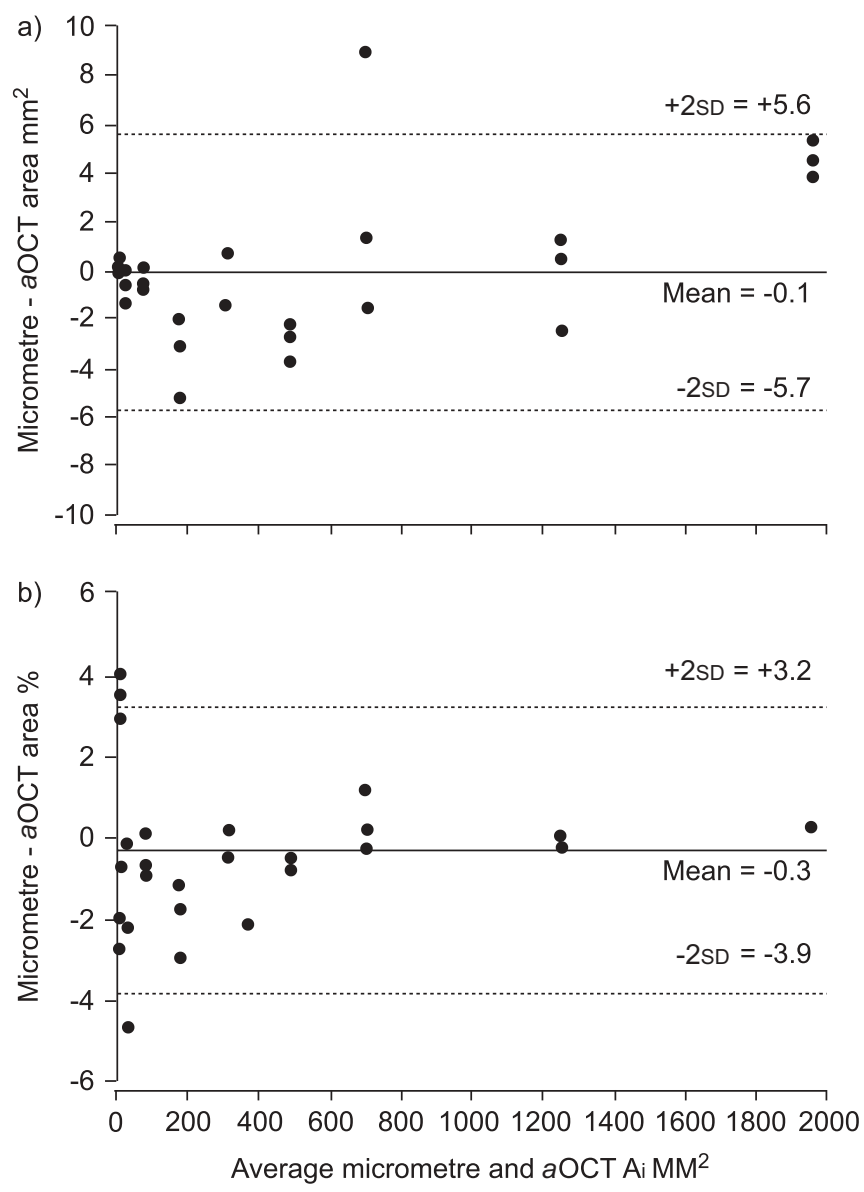

FIGURE 2. Bland-Altman plots of a) absolute and b) relative accuracy of anatomical optical coherence tomography $(\mathrm{aOCT})$ in plastic tubes with internal areas $(\mathrm{Ai})$ in the range $5.3-1,963.5 \mathrm{~mm}^{2}$. Measurements were obtained using an internal micrometer and an aOCT probe.

\section{Anatomical optical coherence tomography}

The $a \mathrm{OCT}$ unit consists of a 1.3-mm wide fibre-optic probe housed within a plastic catheter (outer diameter $2.2 \mathrm{~mm}$ ) as previously described [12-14, 17, 18]. The catheter is passed through the working channel of a standard bronchoscope and into an airway of interest. The probe rotates at $2.5 \mathrm{~Hz}$, directing a broadband light beam (central wavelength $1310 \mathrm{~nm}$, bandwidth $32 \mathrm{~nm}$ ) perpendicular to the airway, thus building up a rotational scan of the internal airway lumen that is displayed on a monitor. The probe is capable of scanning over a radius of $36 \mathrm{~mm}$ with an optimal lateral resolution of $156 \mu \mathrm{m}$ at a distance of $2.35 \mathrm{~mm}$, although this varies with distance. A custom-built computer program enables the user to trace manually the internal lumen and diameter immediately post-acquisition. While rotating, the probe may also be mechanically retracted along an airway segment at $0.4 \mathrm{~mm} \cdot \mathrm{s}^{-1}$, to generate a volumetric dataset. We refer to this as a "pullback" scan.

\section{Plastic tubes}

A plastic airway phantom (online repository, E1) consisting of 10 round tubes (diameters $2.6-50.0 \mathrm{~mm}$ ) was constructed and the tubes were measured using internal micrometers (Bowers Metrology, Bradford, UK) accurate to $0.01 \mathrm{~mm}$. Three separate sites along each tube were imaged by one investigator using 

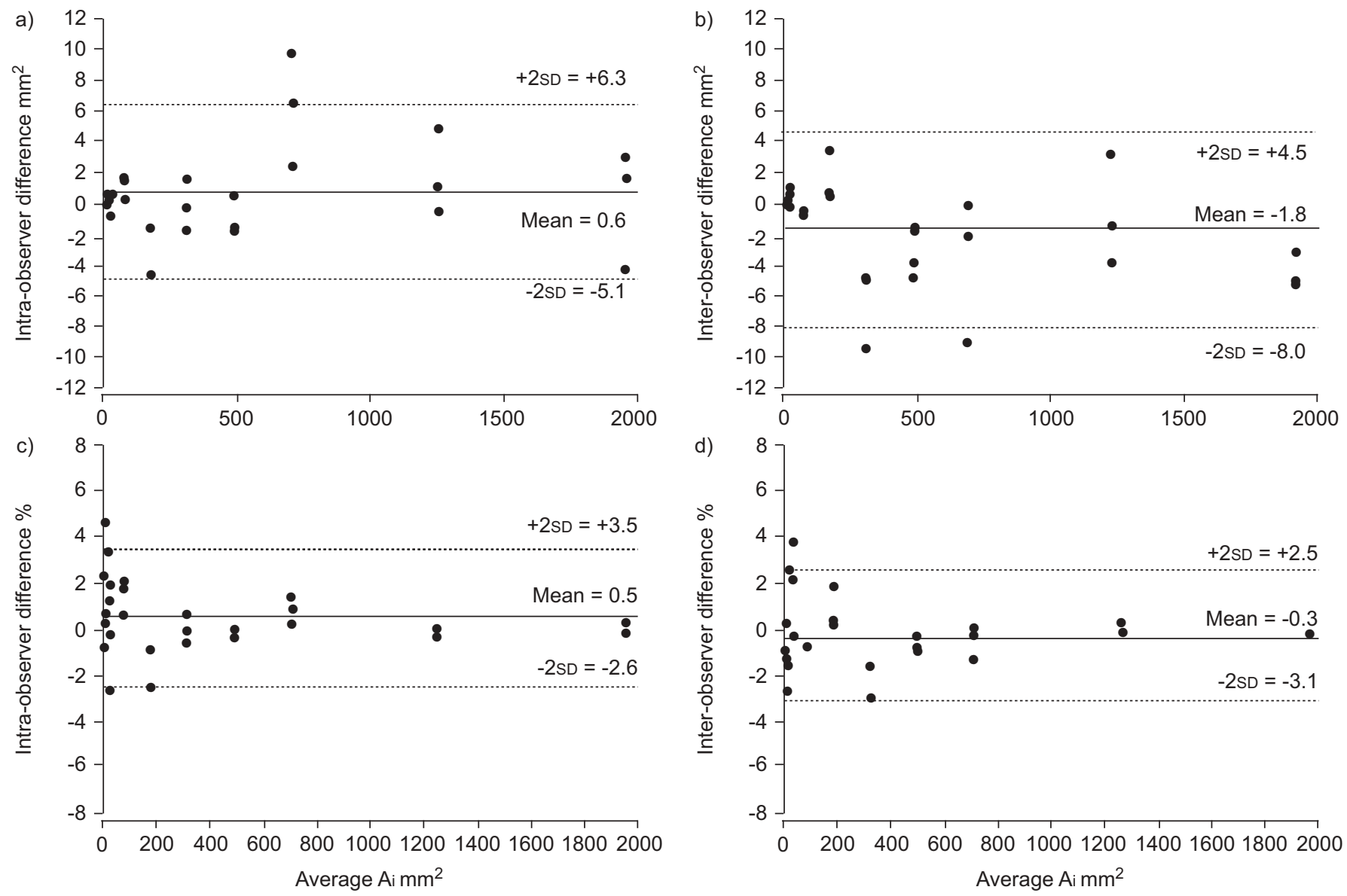

FIGURE 3. Intra-observer (a and c) and inter-observer ( $b$ and d) repeatability Bland-Altman plots for internal lumen area (Ai) using anatomical optical coherence tomography in plastic tubes.

aOCT. The 30 images were then randomised prior to measurement of diameter and internal area $(\mathrm{Ai})$. To evaluate intra-observer repeatability, measurements were repeated after 3 weeks. For inter-observer repeatability, a second investigator repeated the measurements. To assess the effect on measurement accuracy of the bends in the fibre-optic probe during bronchoscopy, in vivo conditions were simulated by repeating the measurements through a bronchoscope inserted into a resuscitation mannequin (online repository, E2).

\section{Excised pig airways}

A trachea and two bronchial segments were dissected from the lungs of an 8-week-old pig. Side branches were ligated with surgical silk and the segments (length $\sim 60 \mathrm{~mm}$ ) were placed horizontally in a perspex chamber of Krebs solution at $37^{\circ} \mathrm{C}$ as described elsewhere [19]. The ends were cannulated, one being connected to a reservoir containing Krebs solution, the height of which could be adjusted to vary the transmural pressure, thereby manipulating airway size (fig. 1).

The airway lumina were visualised using a videoendoscopy technique, whereby a rigid fibre-optic endoscope (SES1711D; Olympus, Tokyo, Japan) was inserted into the airway through an outlet in the organ chamber (fig. 1) [19]. At the site selected for analysis, the mucosal surface was circumferentially stained with dye. Transmural pressures were increased in increments of $5 \mathrm{cmH}_{2} \mathrm{O}$ from $-15 \mathrm{cmH}_{2} \mathrm{O}$ to $+30 \mathrm{cmH}_{2} \mathrm{O}$, then decreased back to $-15 \mathrm{cmH}_{2} \mathrm{O}$. Photographs of the dye-mucosa interface, depicting the inner airway perimeter, were captured and transferred to a computer workstation for analysis of lumen $\mathrm{Ai}$ using ImageJ software, version 1.38t (National Institutes of Health, Bethesda, MD, USA; http://rsb.info.nih.gov/ij). The endoscope was then removed and the aOCT catheter was passed into the airway lumen and advanced to the dye ring, where the measurements were repeated. The order of imaging ( $a$ OCT or endoscope) was randomised. We compared the two techniques by constructing airway compliance curves from the plot of transmural pressure against $\mathrm{Ai}$. Compliance was described first from the slope of the curve between 0 and $10 \mathrm{cmH}_{2} \mathrm{O}$ (the region of maximal change) and secondly by fitting the positive pressure data with an exponential expression (also used to describe the nonlinear elastic behaviour of the lungs), of the form $\mathrm{V}=A-B \times \exp (-K \mathrm{P})$, where $\mathrm{V}$ is airway lumen area, $\mathrm{P}$ is transmural pressure and $A, B$ and $K$ are constants. The Colebatch shape factor $K$ defines the nonlinearity of the area-pressure curve, and is an index of distensibility independent of airway size [20].

To investigate the potential use of $a \mathrm{OCT}$ in the setting of tracheobronchial stenosis, immediately following measurement of airway compliance, a stenosis was simulated in the excised pig trachea by tying suture material around its centre. The $a \mathrm{OCT}$ 

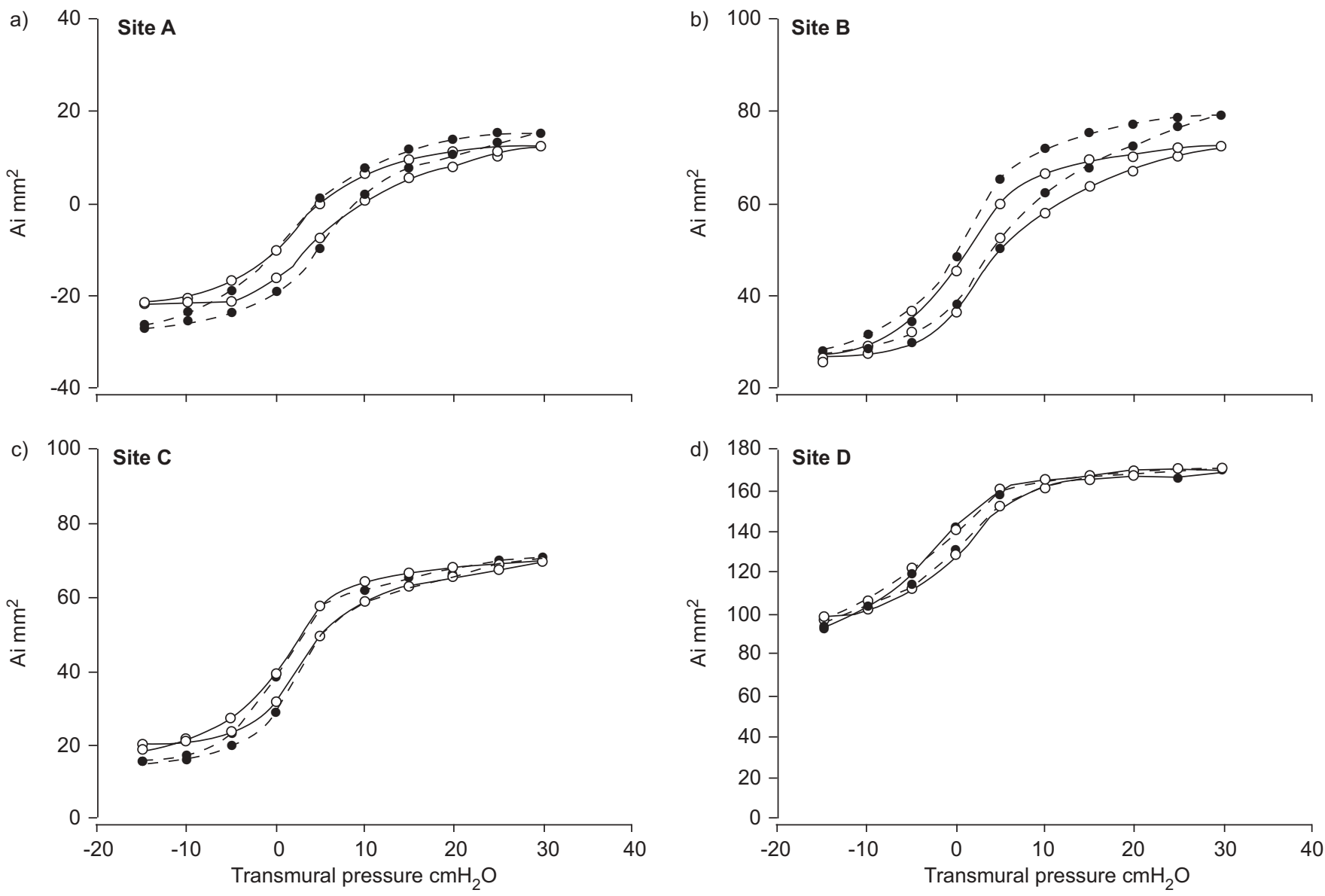

FIGURE 4. Compliance curves in pig airways at four sites (a-d) of differing internal lumen areas (Ai). Compliance was determined by plotting Ai derived from the endoscopic (-- - ) and anatomical coherence tomography $(-)$ techniques against transmural pressure. Comparison of compliance using the Colebatch shape factor $K$ applied to the ascending and descending limbs of the eight curves was not significantly different between the two techniques. Similarly, the mean slope of the curves between $0-10 \mathrm{cmH}_{2} \mathrm{O}$ was not different.

probe was advanced through the tracheal lumen beyond the "stenosis" then mechanically retracted across the stenosis while recording continuously. This pullback scan was analysed using a three-dimensional software viewer (VolView; Kitware, Clifton Park, NY, USA) to measure stenosis dimensions.

\section{In vivo human airways}

Subjects were recruited from patients scheduled for bronchoscopy who also required chest CT as part of their medical care. The 64-slice scanner (Philips Brilliance CT 95089; Philips Medical Systems, Best, The Netherlands) scanned from the glottis to lung bases $(220 \mathrm{~mA}, 120 \mathrm{Kvp}$, voxel size $0.625 \times 0.625 \times 0.625 \mathrm{~mm}$ and using a lung-enhanced reconstruction algorithm). On the same day, a bronchoscopy was performed under propofol anaesthesia titrated to a depth sufficient to permit spontaneous breathing.

To ensure that scans were obtained at a similar lung volume during both the CT and bronchoscopy, impedance plethysmography bands (Respitrace 10.9230; Ambulatory Monitoring, Ardsley, NY, USA) were positioned over the ribcage and abdomen and calibrated using an isovolume manoeuvre. The summed abdominal and ribcage signal is a measure of total chest wall displacement (lung volume change) and was used to monitor lung volume changes during CT and bronchoscopy [21]. During CT scanning, respiration was suspended at functional residual capacity (FRC) using the summed Respitrace signal to guide a voluntary breath-hold. FRC decreases during general anaesthesia [22]. To match FRC during bronchoscopy to the preceding $\mathrm{CT}$, we noted baseline FRC using a period of quiet tidal breathing prior to anaesthetic delivery and, once stable anaesthesia had been achieved, applied positive end expiratory pressure and pressure support to raise FRC back to baseline (online repository, E3). In each subject, between 7 and 11 normal airway sites (from the trachea to the subsegmental airways) were imaged using $a \mathrm{OCT}$ over six respiratory cycles each, taking $\sim 20 \mathrm{~min}$.

After the bronchoscopy, $\mathrm{Ai}$ and short-axis diameter were measured from the a OCT and CT images and compared. CT images were analysed using Pulmonary Workstation 2.0 software (VIDA Diagnostics, Iowa City, IA, USA), which reformats CT data into three-dimensional images and calculates airway area and diameter perpendicular to the airways. At each site, measurements from three adjacent CT slices were averaged. For the aOCT images, measurements were performed at endexpiration (FRC) on three successive breaths and averaged. 


\section{Statistical analyses}

The coefficient of variation of repeated Ai measurements from the plastic phantom was expressed as a percentage. Intra- and inter-observer repeatability coefficients were determined using the Bland and Altman method and a paired t-test compared compliance measurements derived using aOCT with the endoscopic technique [23]. Human airway dimensions measured by CT and aOCT were compared using Bland-Altman plots [23]. Statistical analyses were performed using SPSS for Windows 15.0.0 (SPSS Inc, Chicago, IL, USA).

\section{RESULTS}

\section{Comparative studies}

\section{Plastic tubes}

aOCT-derived Ai measurements in 10 plastic tubes (range 5.3$1,963.5 \mathrm{~mm}^{2}$ ) showed very close agreement to the micrometer Ai measurements (fig. 2), with the mean difference across the tubes being $-0.1 \pm 5.6 \mathrm{~mm}^{2}(-0.3 \pm 3.5 \%)$ with even scatter about the zero difference line. The coefficient of variation of repeated measurements was $0.8 \%$. Measurements repeated through the resuscitation mannequin showed a small bias towards overestimating the $\mathrm{A} i$, and a paired t-test showed the difference of the means to be $2.1 \mathrm{~mm}^{2}$ (95\% confidence interval $0.7-3.5 \mathrm{~mm}^{2}$, $\mathrm{p}<0.01$ ). Bland-Altman plots of intra- and inter-observer variability demonstrated good agreement (fig. 3). The calculated intra- and inter-observer repeatability coefficients were 5.7 and $7.1 \mathrm{~mm}^{2}$, respectively, which represent $<2 \%$ of the mean area of all 10 tubes.

\section{Excised pig airways}

Three airways were dissected and four sites (A-D) were marked with dye (one bronchus was marked at two sites). Airway Ai ranged $9.0-170.0 \mathrm{~mm}^{2}$. At each site, ascending and descending limbs of the pressure/Ai curve were plotted using both endoscope and aOCT-derived Ai (fig. 4). Compliance curves for each technique were similar and the mean shape factor, $K$, was not significantly different (mean $K=0.11$ (endoscopy) versus $\mathrm{K}=0.12 \mathrm{cmH}_{2} \mathrm{O}^{-1}$ (aOCT); 95\% confidence interval for the difference of means -0.03-0.01; $\mathrm{p}=0.24$ ). Compliance measured using the slope of the curve between 0 and $10 \mathrm{cmH}_{2} \mathrm{O}$, was not different between techniques, being 2.2 and $2.1 \mathrm{~mm}^{2} \cdot \mathrm{cmH}_{2} \mathrm{O}^{-1}(95 \%$ confidence interval for the difference of means $-0.3-0.2 ; \mathrm{p}=0.64$ ).

Two- and three-dimensional reconstruction of the tracheal stenosis is shown in figure 5 . At the narrowest point, the transverse dimensions measured $7.9 \times 7.6 \mathrm{~mm}$, with a stenosis length of $23 \mathrm{~mm}$.

\section{In vivo human airways}

$\mathrm{CT}$ and $a \mathrm{OCT}$ measurements were obtained at 36 airway sites from six airway generations in four patients (fig. 6 and online repository, E4). Ai ranged $6.2-282.0 \mathrm{~mm}^{2}$ and short-axis diameters ranged $2.3-16.9 \mathrm{~mm}$. There was close correlation between $\mathrm{CT}$ and $a \mathrm{OCT}$-based $\mathrm{Ai}$ and diameter measurements $(\mathrm{r}=0.99 ; \mathrm{p}<0.001$; online repository, E5). Bland-Altman plots showed aOCT measurements to be slightly greater than CT measurements by a mean of $6.2 \pm 18.1 \mathrm{~mm}^{2}$ for Ai (fig. 7a and b) and $0.4 \pm 1.3 \mathrm{~mm}$ for diameter (fig. 7c and d).
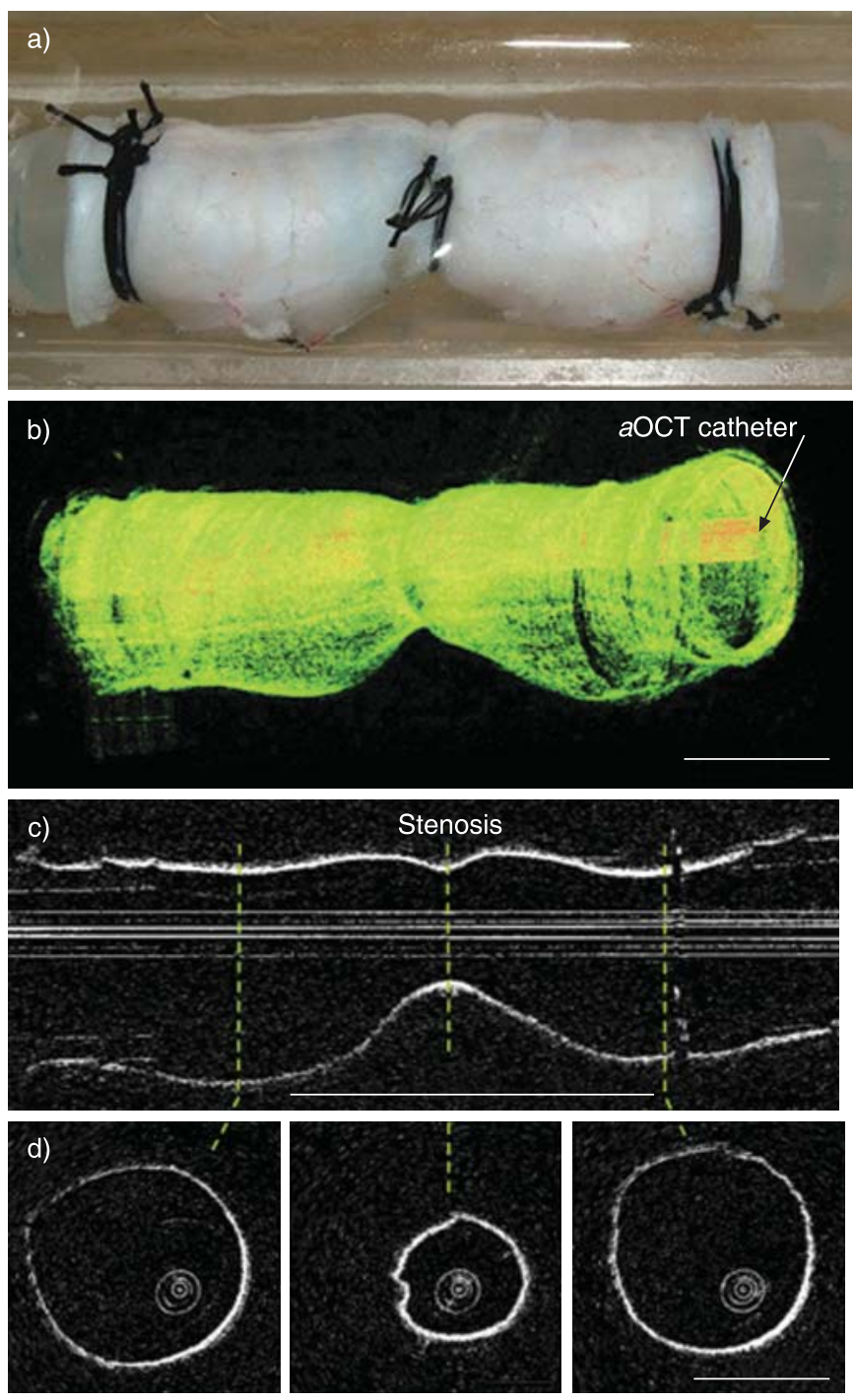

FIGURE 5. a) Simulated stenosis in an excised pig trachea. b) Threedimensional image generated from an anatomical optical coherence tomography "pullback" scan across the stenosis. Scale bar $=10 \mathrm{~mm}$. c) Two-dimensional reconstruction. Scale bar $=23 \mathrm{~mm}$. d) Cross-sections through the stenosis and the normal trachea, from (c), displayed in axial format. Scale bar $=10 \mathrm{~mm}$.

\section{DISCUSSION}

Despite a revolution in the field of thoracic imaging, contemporary bronchoscopists still lack a reliable tool to quantify endobronchial anatomy during a procedure. This paper describes the use of a novel imaging technique, $a \mathrm{OCT}$, in plastic tubes, excised pig airways and in vivo human airways. As aOCT operates independently of conventional bronchoscopic imaging, it overcomes the major limitation of bronchoscopic airway quantification: distorted images from which airway dimensions are difficult to derive, requiring postprocedure analysis. The results of our validation studies indicate that $a \mathrm{OCT}$ is a suitable complementary bronchoscopic technique for real-time measurements of airway dimensions.

This study demonstrates the utility and accuracy of $a \mathrm{OCT}$ in three experimental settings. In plastic tubes, $a \mathrm{OCT}$ provided accurate and repeatable Ai measurements over a wide range of 

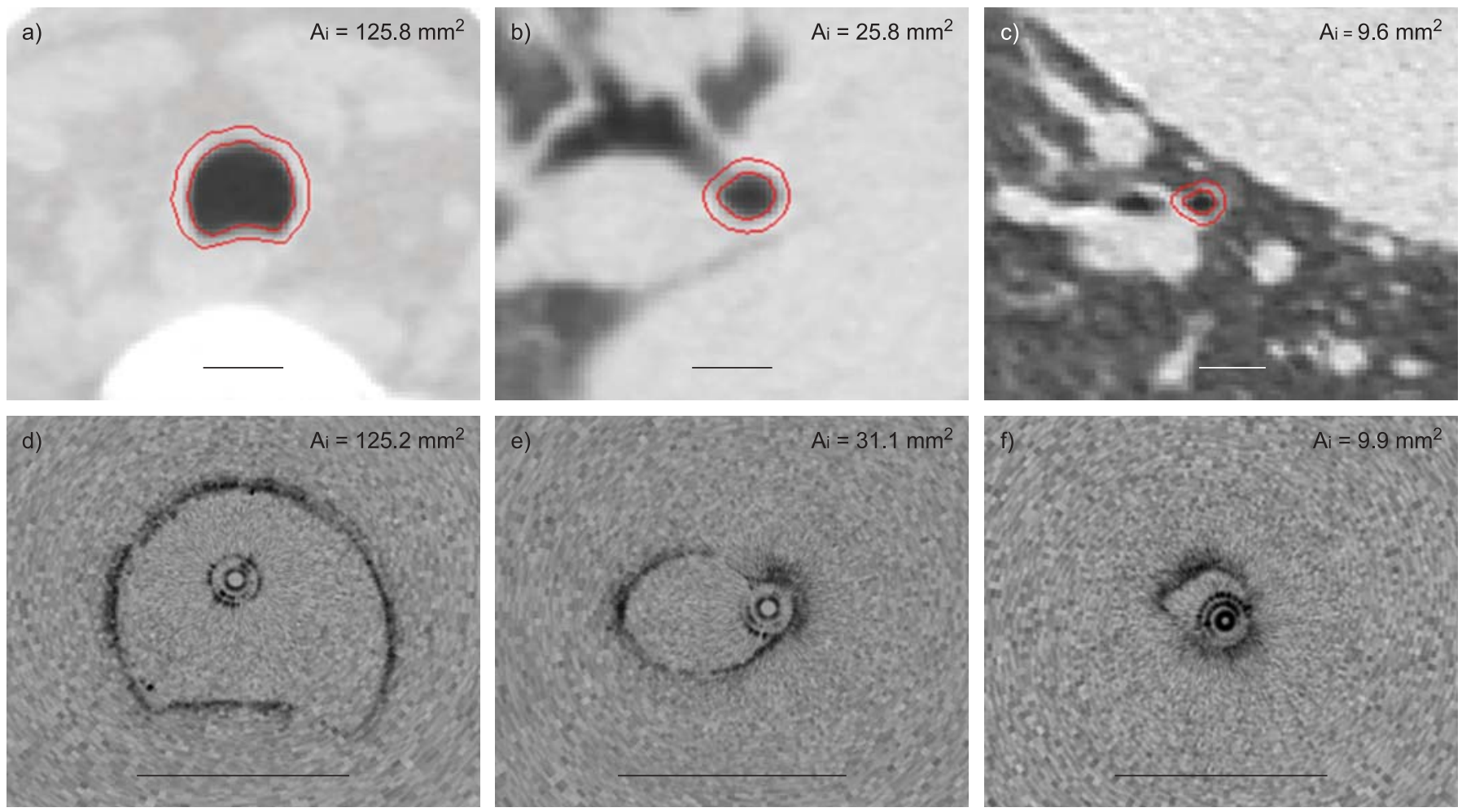

FIGURE 6. Computed tomography (CT) images (a-c) and their corresponding anatomical optical coherence tomography images (d-f) at: a) proximal trachea; b) left lower lobe; and c) medial segment of the right middle lobe bronchus. The CT images, taken at functional residual capacity, demonstrate the internal and external airway wall perimeter as calculated using Pulmonary Workstation 2.0 software (VIDA Diagnostics, lowa City, IA, USA). Internal lumen area (Ai) measurements are shown for each technique. Scale bars $=10 \mathrm{~mm}$.

diameters. Relative to micrometer measurements, Ai measured when the $a \mathrm{OCT}$ catheter was passed through a mannequin was greater, on average, by $2.1 \mathrm{~mm}^{2}$. This may be due to bends imposed on the catheter/probe during in vivo scanning, causing torsion and uneven probe rotation. Regardless, the magnitude of this difference (representing $<1 \%$ of the mean area of the 30 measurements) is clinically insignificant. Compliance measurements in excised pig airways using $a \mathrm{OCT}$ and an endoscopic technique were also similar, highlighting the potential for $a \mathrm{OCT}$ to determine physiological properties of airways, such as regional compliance during bronchoscopy. Lastly, comparison of $a \mathrm{OCT}$ with CT measurements in humans also showed close agreement. A strength of our approach was that great care was taken to control subject lung volume, which can affect airway size [24]. Indeed, to our knowledge, among quantitative videobronchoscopy studies, this is the first such study to do so. However, while every attempt was made to standardise conditions during CT and $a \mathrm{OCT}$ scanning, the measurements were performed several hours apart, making comparison of the exact site within the airway difficult. Simultaneous imaging would have been preferable, but this would be difficult to achieve as simultaneous bronchoscopy and CT scanning is complex and would cause CT image artefact.

While, as discussed above, there was close agreement between $a \mathrm{OCT}$ and CT, overall, $a \mathrm{OCT}$ yielded measurements of diameter and $\mathrm{Ai}$ that were $7.6 \%$ and $15.1 \%$ greater than those produced CT measurements, respectively. This finding contrasts with the study by Coxson et al. [25] who, using conventional OCT, found measurements of $\mathrm{Ai}$ in small airways (mean airway size $12.5 \pm 6.1 \mathrm{~mm}^{2}$ ) to be lower when measured with OCT than with CT, by an average of $31 \%$. The discrepancy is readily attributable to the higher CT lung volumes in the study of CoxsON et al. [25] (total lung capacity) relative to their bronchoscopic OCT images (tidal breathing during conscious sedation). With the use of impedance plethysmography bands, our study carefully controlled lung volume, allowing us to estimate FRC during CT scanning and approximate this lung volume during bronchoscopy.

We found the relative difference between $\mathrm{CT}$ and aOCT measurements to be greatest in smaller airways (fig. $7 \mathrm{c}$ and $\mathrm{d}$ ). We have identified two mechanisms for this disparity. Firstly, it is possible that the presence of the aOCT catheter could mechanically increase small airway calibre, leading to an overestimation of $\mathrm{Ai}$, although we measured only airways larger than the catheter with a partial air space evident between the two (fig. 6f). Secondly, CT partial volume averaging, which results in underestimation of $\mathrm{A} i$, is particularly evident in airways $<2-3 \mathrm{~mm}$ in diameter and increases with the obliqueness of the imaging plane [26, 27]. Also, partial volume averaging may have been more significant in our study as we scanned at FRC rather than total lung capacity. This is less of a concern with $a \mathrm{OCT}$, where the "slice thickness" equivalence of an axial $a$ OCT image in, for example, a 5-mm diameter airway is $\sim 0.2 \mathrm{~mm}$, compared with $0.625 \mathrm{~mm}$ using modern CT scanners. Further, unlike CT, the $a \mathrm{OCT}$ probe lies within the plane of the 

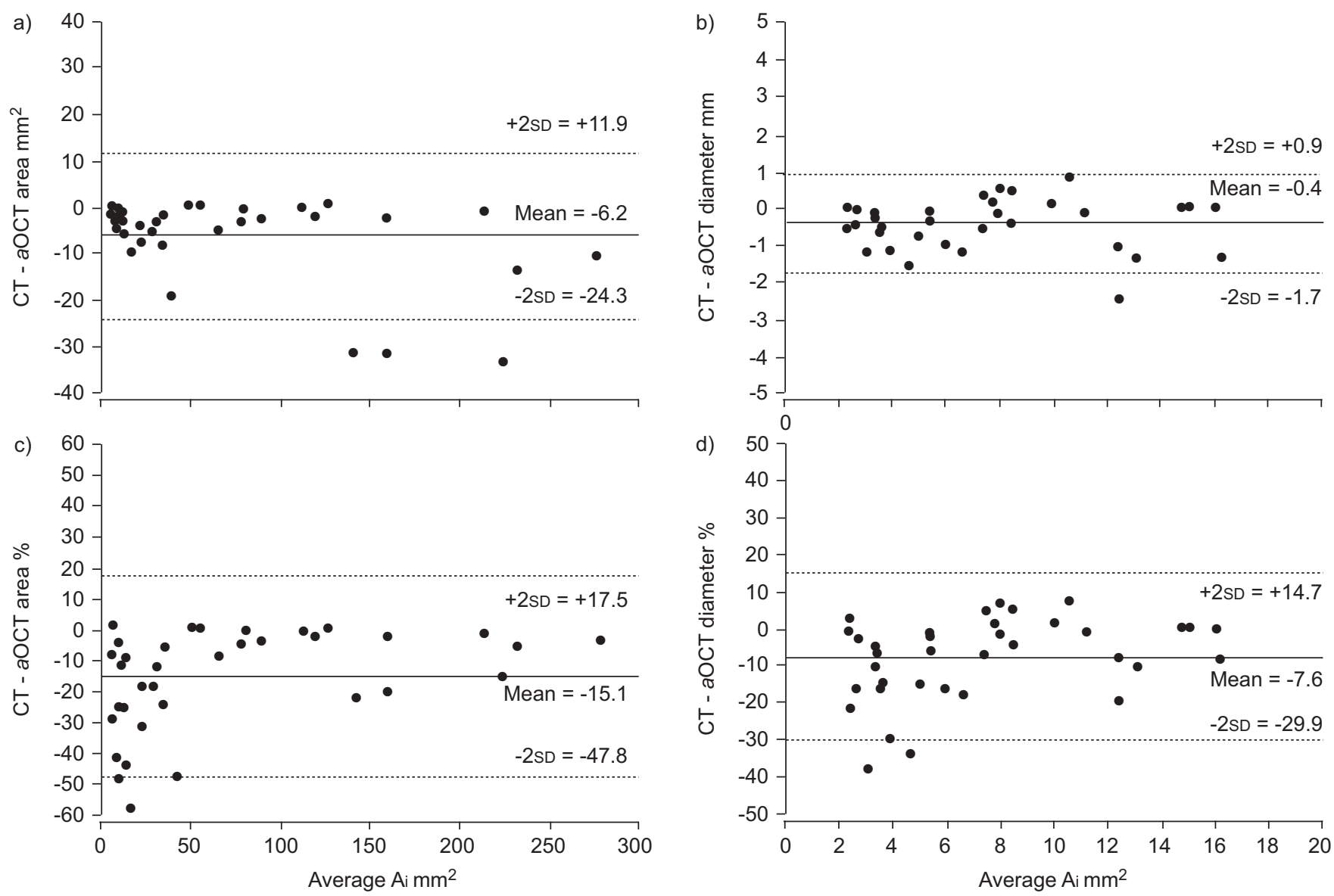

FIGURE 7. Bland-Altman plots of absolute ( $\mathrm{a}$ and $\mathrm{b}$ ) and percent ( $\mathrm{c}$ and $\mathrm{d}$ ) measurement differences comparing human airway area (Ai) and short-axis diameter using computed tomography (CT) and anatomical optical coherence tomography (aOCT)

airway and scanning occurs close to the airway axis, avoiding oblique imaging. Even in the largest airways, in which the relative size of the bronchoscope to airway lumen increases the likelihood of oblique scanning, an angle approaching $18^{\circ}$ would be required to produce a $5 \%$ area error (online repository, E6).

\section{Potential applications}

$a$ OCT could prove beneficial in several bronchoscopic settings. The deployment of stents for airway stenoses requires prebronchoscopy imaging, usually with CT. This provides important information about the site, number and dimensions of stenoses. However, CT is usually performed at total lung capacity and scans are not always recent at the time of procedure. It is still, therefore, incumbent upon the bronchoscopist to estimate stenosis length and calibre, which can be highly subjective. The ability to scan a stenosis and derive immediate quantitative dimensions would be highly advantageous for stent size selection. By virtue of real-time imaging, aOCT could also prove useful in the management of the malacia disorders, where quantitative assessment of dynamic airway collapse is desirable. This may be particularly useful in the setting of paediatric bronchology, where longitudinal assessment of tracheo-bronchomalacia is required, ideally without ionising radiation. Finally, measurement of airway size is required during endobronchial valve deployment for endoscopic lung volume reduction. This relatively new field might benefit from the capacity of aOCT to size airway diameters optimising valve selection.

\section{Limitations}

Although $a$ OCT can image a wide range of airway sizes, the current prototype has limited subsurface detail and, therefore, cannot measure airway wall thickness. The catheter size could also be considered a limitation as the probe-catheter system limits the size of measureable airways to $\sim 2.5 \mathrm{~mm}$. This gets close to but does not image the small airways of greatest interest in asthma and chronic obstructive pulmonary disease. However, this technical limitation is temporary as smaller probes are in development.

Probe rotation speed was found to be adequate for scanning during normal breathing. Faster acquisition speeds would improve the capacity for three-dimensional reconstructions to account for respiratory and cardiac motion. Despite this, we have recently demonstrated the capacity to "gate" images to specific phases of the respiratory cycle [28]. Finally, as with any new technology, cost is a factor. Our device is a custom-built prototype, making a unit cost difficult to estimate. Larger centres specialising in interventional procedures will probably be the first to realise the potential of and to benefit from this technique. 


\section{Conclusion}

This paper demonstrates the accuracy and precision of $a \mathrm{OCT}$ in phantom, pig and human airways and demonstrates its capacity to reconstruct three-dimensional images. An advantage of this technique over existing methods of bronchoscopic airway quantification is the ability to operate independent of the distorting effects of the bronchoscope. Such a tool could be of considerable value to interventional bronchoscopists, by improving assessment of disease severity and its progression, sizing dimensions of airway stenoses in preparation for stenting and for the deployment of endobronchial valves. Future studies examining the effectiveness of $a \mathrm{OCT}$ in these and other clinical settings will further define the optimal role of this emerging technique.

\section{SUPPORT STATEMENT}

This study was supported by an Australian National Health and Medical Research Council (NHMRC; Canberra, Australia) Project Grant No. 513854. J.P. Williamson is funded by a NHMRC Postgraduate Research Scholarship No. 463926, a Sir Charles Gairdner Hospital (Perth, Australia) Research Grant and a University of Western Australia (Perth, Australia) top-up grant. P.R. Eastwood is funded by a NHMRC Senior Research Fellowship No. 513704. R.A. McLaughlin is funded by the Raine Medical Research Foundation (Nedlands, Australia). P.B. Noble is funded by an Australian-based Biomedical Fellowship, No. 513921.

\section{STATEMENT OF INTEREST}

Statements of interest for J.P. Williamson, J.J. Armstrong, R.A. McLaughlin, M.J. Phillips, D.D. Sampson, D.R. Hillman and P.R. Eastwood can be found at www.erj.ersjournals.com $/ \mathrm{misc} /$ statements.dtl

\section{ACKNOWLEDGEMENTS}

The authors wish to acknowledge the enthusiastic support of N. Hicks, P. Muir and C. Smith from the Radiology Dept of Sir Charles Gairdner Hospital (SCGH; Perth, Australia). They also wish to thank C. Smith and M. Schmidt from the Dept of Anaesthesia, SCGH, for their anaesthetic expertise and J. Graham and W. Lilly (SCGH) for assistance with the mannequin study. The authors are sincerely grateful to R. Kap and T. Deans from the Dept of Medical Technology and Physics, SCGH, for assistance with construction of the airway phantom and technical support and to B. Manners from the Audiovisual Production Unit, SCGH, for illustration support.

\section{REFERENCES}

1 Ferretti GR, Kocier M, Calaque O, et al. Follow-up after stent insertion in the tracheobronchial tree: role of helical computed tomography in comparison with fiberoptic bronchoscopy. Eur Radiol 2003; 13: 1172-1178.

2 Wadsworth SJ, Juniper MC, Benson MK, et al. Fatal complication of an expandable metallic bronchial stent. Br J Radiol 1999; 72: 706-708.

3 Doolin EJ, Strande LL. Calibration of endoscopic images. Ann Otol Rhinol Laryngol 1995; 104: 19-23.

4 Forkert L, Watanabe $\mathrm{H}$, Sutherland $\mathrm{K}$, et al. Quantitative videobronchoscopy: a new technique to assess airway caliber. Am J Respir Crit Care Med 1996; 154: 1794-1803.

5 McFawn PK, Forkert L, Fisher JT. A new method to perform quantitative measurement of bronchoscopic images. Eur Respir J 2001; 18: 817-826.

6 Santos MC, Strande L, Doolin EJ. Airway measurement using morphometric analysis. Ann Otol Rhinol Laryngol 1995; 104: 835-838.

7 Dorffel WV, Fietze I, Hentschel D, et al. A new bronchoscopic method to measure airway size. Eur Respir J 1999; 14: 783-788.
8 Czaja P, Soja J, Grzanka P, et al. Assessment of airway caliber in quantitative videobronchoscopy. Respiration 2007; 74: 432-438.

9 Masters IB, Eastburn MM, Wootton R, et al. A new method for objective identification and measurement of airway lumen in paediatric flexible videobronchoscopy. Thorax 2005; 60: 652-658.

10 Hanna N, Saltzman D, Mukai D, et al. Two-dimensional and 3dimensional optical coherence tomographic imaging of the airway, lung, and pleura. J Thorac Cardiovasc Surg 2005; 129: 615-622.

11 Whiteman SC, Yang Y, van Pittius DG, et al. Optical coherence tomography: real-time imaging of bronchial airways microstructure and detection of inflammatory/neoplastic morphologic changes. Clin Cancer Res 2006; 12: 813-818.

12 Armstrong JJ, Leigh MS, Walton ID, et al. In vivo size and shape measurement of the human upper airway using endoscopic longrange optical coherence tomography. Opt Express 2003; 11: 1817-1826.

13 Armstrong JJ, Leigh MS, Sampson DD, et al. Quantitative upper airway imaging with anatomic optical coherence tomography. Am J Respir Crit Care Med 2006; 173: 226-233.

14 Leigh MS, Armstrong JJ, Paduch A, et al. Anatomical optical coherence tomography for long-term, portable, quantitative endoscopy. IEEE Trans Biomed Eng 2008; 55: 1438-1446.

15 Walsh JH, Leigh MS, Paduch A, et al. Effect of body posture on pharyngeal shape and size in adults with and without obstructive sleep apnea. Sleep 2008; 31: 1543-1549.

16 Walsh JH, Leigh MS, Paduch A, et al. Evaluation of pharyngeal shape and size using anatomical optical coherence tomography in individuals with and without obstructive sleep apnoea. J Sleep Res 2008; 17: 230-238.

17 McLaughlin RA, Williamson JP, Phillips MJ, et al. Applying anatomical optical coherence tomography to quantitative 3D imaging of the lower airway. Opt Express 2008; 16: 17521-17529.

18 Williamson JP, McLaughlin RA, Phillips MJ, et al. Using optical coherence tomography to improve diagnostic and therapeutic bronchoscopy. Chest 2009; 136: 272-276.

19 Mitchell H, Sparrow M. Video-imaging of lumen narrowing; muscle shortening and flow responsiveness in isolated bronchial segments of the pig. Eur Respir J 1994; 7: 1317-1325.

20 Colebatch $\mathrm{HJH}, \mathrm{Ng} \mathrm{CKY}$, Nikov N. Use of an exponential function for elastic recoil. J Appl Physiol 1979; 46: 387-393.

21 Konno K, Mead J. Measurement of the separate volume changes of rib cage and abdomen during breathing. J Appl Physiol 1967; 22: 407-422.

22 Wahba RWM. Perioperative functional residual capacity. Can J Anaesth 1991; 38: 384-400.

23 Bland JM, Altman DG. Statistical methods for assessing agreement between two methods of clinical measurement. Lancet 1986; 1: 307-310.

24 Brown RH, Scichilone N, Mudge B, et al. High-resolution computed tomographic evaluation of airway distensibility and the effects of lung inflation on airway caliber in healthy subjects and individuals with asthma. Am J Respir Crit Care Med 2001; 163: 994-1001.

25 Coxson HO, Quiney B, Sin DD, et al. Airway wall thickness assessed using computed tomography and optical coherence tomography. Am J Respir Crit Care Med 2008; 177: 1201-1206.

26 Dame Carroll JR, Chandra A, Jones AS, et al. Airway dimensions measured from micro-computed tomography and high-resolution computed tomography. Eur Respir J 2006; 28: 712-720.

27 Williamson JP, James AL, Phillips MJ, et al. Quantifying tracheobronchial tree dimensions: methods, limitations and emerging techniques. Eur Respir J 2009; 34: 1-14.

28 McLaughlin RA, Armstrong JJ, Becker S, et al. Respiratory gating of anatomical optical coherence tomography images of the human airway. Opt Express 2009; 17: 6568-6577. 